(C) 1980. The Genetical Society of Great Britain

\title{
PATTERN COMPENSATION IN DROSOPHILA WING VEIN DEVELOPMENT
}

\author{
JAMES N. THOMPSON, Jr., JOE V. TONEY and G. BRADLEY SCHAEFER \\ Department of Zoology, University of Oklahoma, Norman, Oklahoma 73019
}

Received 31.vii.79

\section{SUMMARY}

Much interest in developmental genetics has focused on the formation of patterns. Using the wing vein pattern of Drosophila melanogaster as our model system, we show that vein placement is essentially independent of cell lineage. In studying vein placement in a variety of wing vein mutants, we observed that the position of a vein can move to compensate for the loss of vein in nearby parts of the wing. These observations led us to consider an interacting diffusion gradient model of vein formation.

\section{INTRODUGTION}

THE sophisticated methods of molecular and developmental genetics have given us detailed insights into the mechanisms of cellular differentiation. One major problem still facing developmental genetics, however, is the way in which spatial information is genetically determined. A number of hypotheses have been put forward to explain pattern formation (e.g., Stern, 1968; Wolpert, 1969; Lawrence, 1973; Kauffman, 1977; Garcia-Bellido and Ripoll, 1978; Tokunaga, 1978), many involving some form of diffusion gradient to code positional information. According to such models, cells differentiate in response to positional information based upon their genetic competence.

Pattern formation can be studied in a variety of simple model systems, such as the arrangement of wing veins in Drosophila melanogaster. The vein pattern has a number of advantages, since many vein mutations have been described and one can dissect, culture, and carry out mosaic and histochemical studies with the imaginal wing disk in larvae and pupae. Our experience with polygenic variation and the response of patterns to artificial selection has allowed us to use this model system to study mechanisms of pattern formation in several unique ways. Our basic approach has been to identify and quantify minor pattern changes that occur in response to the introduction of well-defined vein mutations or to controlled manipulation of the polygenic modifiers of vein expression.

The variation that is detectable in vein pattern led us to study the developmental basis of vein formation. Cell lineage in the growing wing disk results in clones that are generally longitudinal and parallel to the adult veins (Bryant, 1970). Thus, one possibility is that each vein is the product of a lineage of "vein-determined" cells. A brief consideration of the behaviour of large clones produced by the Minute technique of GarciaBellido et al. (1973), however, showed that this can not be the case (Thompson, unpublished; Schaefer and Thompson, 1977). The unusually large clones 
generated by this technique are the result of an overgrowth by wild type cells in a more slowly dividing Minute cell background. If the original cell of a non-Minute clone were determined to be " non-vein", one would expect an absence, or at least a major disruption, of the vein pattern in the clone. A similar prediction might be made for an original cell determined to be "vein". No such disruption is seen. The vein pattern in these large clones is completely normal. Thus, it is unlikely that the veins per se are determined by cell lineage, although we shall argue later that it is reasonable to propose that some prepattern of vein capacity is related to cell lineage.

In turning our attention to tissue-level mechanisms we were struck by a number of vein interactions that were consistent with a diffusion gradient model of vein determination (Schaefer, Toney, and Thompson, 1978). In this paper we shall first summarise data which confirms that the veins are, at least to a major extent, independent of cell lineage in the wing. We shall then describe evidence supporting a general model of interacting diffusion gradients which might explain the origin of vein patterns and the homeostasis required to compensate for natural variations in wing shape and size.

\section{MATERIALS AND METHOdS}

All flies were maintained on an agar, yeast, cornmeal, and molasses medium at $25 \pm 1{ }^{\circ} \mathrm{C}$. Stocks used in this study included three wild type strains (OK1, Woodruff and Thompson, 1977; Canton-S; and Oregon-R), selected lines of radius incompletus $(r i, 3-47 \cdot 0)$ in which the second longitudinal vein (L2) is shortened, and two stocks of telegraph of Carlson $\left(\operatorname{tg}^{C}, 2-0 \cdot 0\right.$, Thompson, 1973). One stock of $\operatorname{tg}^{C}$ was a selected line in which the L2 vein is completely absent; the other strain was unselected. Mosaic analyses were done using plexus ( $p x, 2-100 \cdot 5)$, multiple wing hairs (mwh, 3-0.0), veinlet (ve, 3-0.2), and Minute(3) $55(M(3) 55,3-28 \cdot 9$, Garcia-Bellido, 1977). A description of these analyses is included in the results section. See Lindsley and Grell (1968) for a description of mutants.

In order to quantify intervein distances, slides were made of wings sampled from female flies of each stock. From the varying phenotypes of the unselected $\operatorname{tg}^{C}$ stock, we measured those flies with a complete L2 vein. Photographs were taken of each wing and distances were measured from the prints. All intervein distances were standardised to wing size. Counts of marginal wing hairs were made directly from the slides.

\section{Results}

\section{(i) Mosaic analysis}

Garcia-Bellido and his colleagues (1975; Garcia-Bellido, et al., 1973) reported that large marked clones do not cross a boundary separating the anterior and posterior halves (compartments) of the Drosophila wing. This compartment boundary is essentially straight and lies several cell diameters anterior to the L4 vein. Thus, in an attempt to understand the relationship between vein formation and cell lineage (as reflected in the establishment of wing compartments), we generated large clones in wings homozygous for vein gap mutants or for mutants that altered the relative position of the L4 vein (see Schaefer and Thompson, 1977).

The recessive mutant veinlet (ve) produces terminal gaps in all longitudinal 

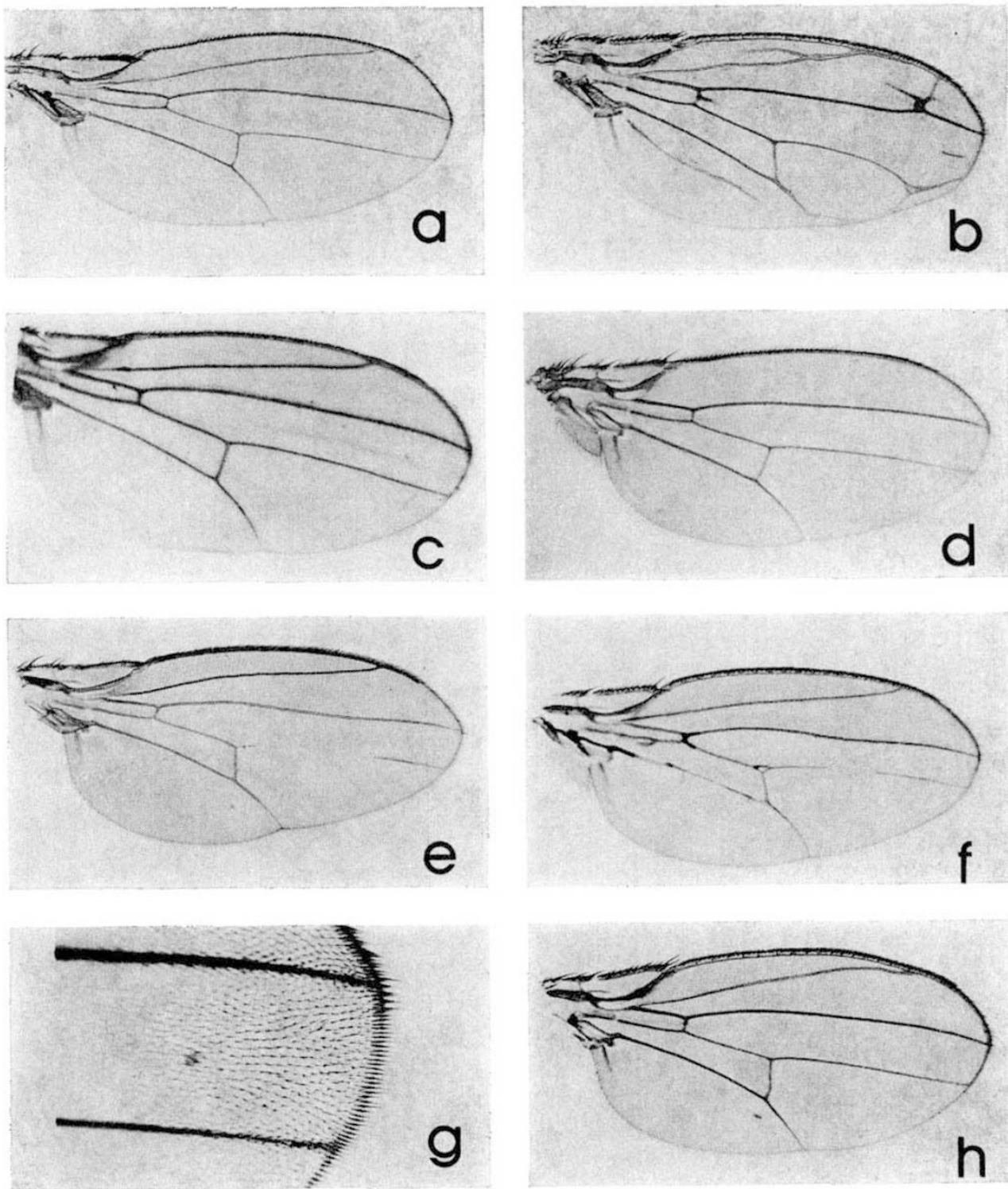

FIG. 1.-Wings of Drosophila melanogaster. $a$, wild type; $b$, plexus; $c$, telegraph of Carlson with entire L2 present; d, telegraph of Carlson Short (selected for absence of L2 vein); $e, f$, cubitus interruptus; $g$, wing margin showing hairs between the $\mathrm{L} 3$ and $\mathrm{L} 4$ veins; $h$, radius incompletus Long. 
veins. Large clones were generated in ve wings by irradiating 0-72 hour mwh $v e+1+v e M(3) 55$ eggs and larvae with $1000 \mathrm{r}$. Somatic recombination produces homozygous mwh $v e+$ cells that grow at a relatively faster rate than the surrounding non-mwh cells that are heterozygous for $M(3) 55$ (Garcia-Bellido et al., 1973). Ten per cent (36 of 369) of the ve wings had mwh clones. Of these, 17 were large, running along part or all of the compartment boundary ( 10 of the 17 extended along the distal boundary to the margin of the wing in the area affected by $v e$ ). None showed a change in compartment position, in spite of the wide variation in L4 vein length.

The mutants plexus $(p x)$ and net (net) add fragments of extra vein and cause a change in position of some veins, including the distal region of the L4 (figure 1). If the shift in the position of the L4 in plexus and net is associated with changes in cell lineage or cell death, one might expect to see it reflected in the position of the compartment boundary at the tip of the wing. We found that, although there were some minor variations, the position of the compartment boundary was not altered in these mutants. For example, in $825 p x$ wings heterozygous for $m w h$ and $M(3) 55,89$ clones of various sizes were found. Of these, 47 ran along part or all of the compartment boundary, and two instances were found where the clone appeared to cross the boundary. Among 222 net wings scored, four out of a total of 33 clones were found to meet the boundary, but none crossed it.

In both $p x$ and net, posterior clones occasionally dipped to follow the displaced L4. This, however, is only a movement within the posterior compartment and does not involve crossing a boundary. The margin of large clones along the compartment boundary was often jagged, and the two instances in which a clone appeared to disregard the boundary in $p x$ did not occur in the distal area where the L4 vein is displaced. In other words, there is no consistent change in the compartment boundary correlated with alterations in the placement of the L4 vein. It is therefore most likely that the examples of a jagged or apparently crossed compartment boundary are simply associated with cell death or some other process acting between the anterior and posterior compartments in these mutants.

These studies of large mosaic clones tend to support the hypothesis that vein determination is essentially independent" of cell lineage, at least in so far as it is reflected by the separation of the wing into anterior and posterior compartments. On the other hand, one might still argue that veins are associated with regional lineages of vein-competent cells within compartments. As discussed in the introduction, however, the absence of disrupted vein patterns in the large non-Minute clones leads one to believe that vein placement is not directly correlated with precise lineages of vein-competent cells.

\section{(ii) Pattern compensation}

The principal tissue-level mechanisms proposed for the coding of developmental responses often involve diffusion of some cell product or products. The part which diffusion is thought to play in development, however, varies greatly from system to system. One of the most striking things about the arrangement and spacing of veins in the adult Drosophila wing is that they are long and fairly straight, and the L3 and L4 veins are formed parallel to each other throughout a major part of their length. This suggests that the placement of one vein might be dependent upon the placement of other veins, 
For example, vein spacing could theoretically be the product of an interaction between diffusion gradients initiated along the margin of the developing wing blade. In this section we shall describe the experimental evidence that tends to support such a model.

Indirect evidence for diffusion activity in wing vein development came from a survey of vein mutants and selection lines. One dramatic example of vein interaction is seen between the L3 and L4 veins in cubitus interruptus (ci) (fig. 1). The L4 vein of $c i$ has a subterminal gap that varies in size. In wings with a moderate size gap, the L3 vein shows a marked bend toward the gap in the L4. Indeed, the dimensions of the L3 bend and of the L4 gap are the same, as a comparison of wings with different size gaps shows (fig. 1). Similar compensations have been observed to affect other vein pairs, such as the L2 in the L3 vein mutant tilt.

A selected line of an L2 vein mutant allowed us to look for spacing compensations involving whole veins, rather than parts of veins. The recessive mutant telegraph of Carlson $\left(\mathrm{tg}^{\mathrm{C}}\right)$ is characterised by proximal L2 gaps, and a selected line ( $\operatorname{tg}^{C}$ Short) is available in which the entire L2 vein is
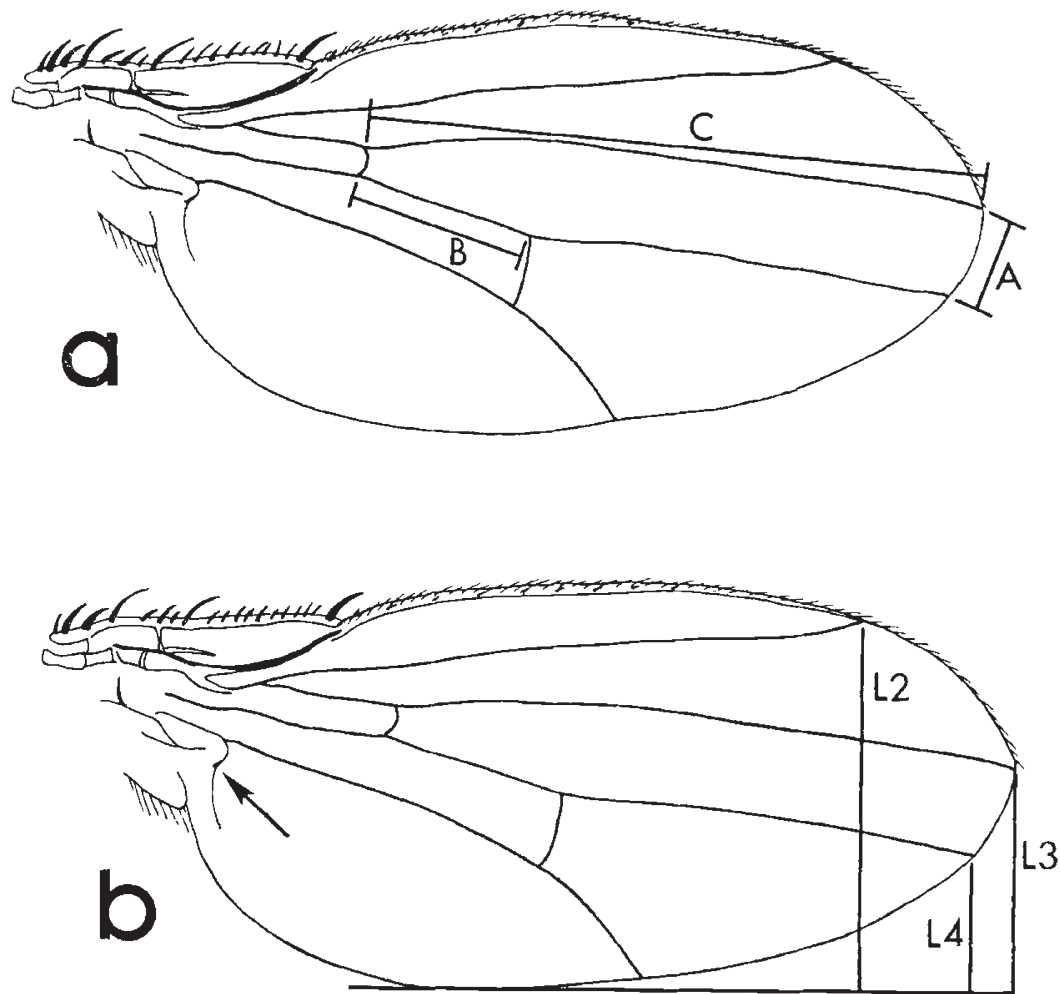

Fig. 2.-Distances measured to quantify vein spacing. a, measurements used to standardise the separation of the L3 and L4 veins with respect to wing length; $b$, measurements used to standardise vein placement with respect to wing width. The L2, L3, and L4 distances are measured from a base line drawn parallel to a line intersecting the L6 hinge area (marked with an arrow) and the distal tip of the L3. Wing width was measured as the greatest distance perpendicular to the base line. 
absent (c.f. Carlson, 1966, 1970). The measurements used to quantify vein spacing are shown in fig. 2a. The data are summarised in table 1.

TABLE 1

Vein pattern measurements and ratios for three lines carrying mutants that affect $L 2$ vein expression and for three wild type control stocks. (Means and standard deviations are given for $A, B$, and $C$ distances shown in fig. $2 ; \mathcal{N}=$ sample size; $P$-values for all comparisons are shown in table 2)

\begin{tabular}{|c|c|c|c|c|c|c|c|}
\hline Stock & $\mathbf{N}$ & Length A & Length B & Length $\mathrm{C}$ & $\mathrm{A} / \mathrm{B}$ & $\mathrm{B} / \mathrm{C}$ & $D^{*}$ \\
\hline $\operatorname{tg}^{C}$ Short & 50 & $14 \cdot 0 \pm 0 \cdot 77$ & $25 \cdot 1 \pm 1 \cdot 43$ & $93 \cdot 9 \pm 4 \cdot 06$ & 0.558 & $0 \cdot 268$ & $2 \cdot 091$ \\
\hline $\operatorname{tg}^{C}$ unselected & 20 & $14.5 \pm 0.96$ & $30 \cdot 1 \pm 2 \cdot 79$ & $103.7 \pm 3.99$ & 0.486 & $0 \cdot 290$ & $1 \cdot 694$ \\
\hline$r i$ Short & 20 & $14.2 \pm 0.82$ & $29 \cdot 4 \pm 2 \cdot 34$ & $103 \cdot 9 \pm 7 \cdot 12$ & $0 \cdot 486$ & $0 \cdot 283$ & $1 \cdot 742$ \\
\hline$O K 1$ & 50 & $12 \cdot 7 \pm 0 \cdot 88$ & $27 \cdot 2 \pm 2 \cdot 51$ & $96 \cdot 3 \pm 5 \cdot 58$ & 0.471 & $0 \cdot 282$ & $1 \cdot 672$ \\
\hline Canton-S & 20 & $13 \cdot 4 \pm 0 \cdot 80$ & $29 \cdot 2 \pm 1 \cdot 63$ & $107 \cdot 4 \pm 5 \cdot 86$ & $0 \cdot 458$ & $0 \cdot 272$ & $1 \cdot 684$ \\
\hline Oregon- $R$ & 20 & $16.4 \pm 0.57$ & $29 \cdot 2 \pm 1 \cdot 62$ & $106 \cdot 0 \pm 4 \cdot 70$ & 0.565 & $0 \cdot 275$ & $2 \cdot 057$ \\
\hline
\end{tabular}

* This is the ratio of the two partly-standardised ratios, $A / B$ and $B / C$.

Since the L2 vein is absent in $\operatorname{tg}^{C}$ Short, we would predict that the L3 vein would be shifted away from the $L 4$ vein if pattern compensation occurs. An increase in distance " A " relative to the size of the wing would represent such a shift in the L3. In order to correct for variations in wing size, we chose the distance from the anterior to the posterior crossveins (B) as an internal standard. This small measurement has several advantages over simple wing length, including the fact that it provided us with a ratio of convenient magnitude. The data support our prediction; the distance A/B in $\operatorname{tg}^{C}$ Short is significantly larger $(\mathbf{P}<0.001)$ than that in the wild type control wings, Canton $S$ and $O K 1$.

In order to improve our standard for wing size, we corrected B for variations in wing size $(B / C)$ and derived the final measure " $D$ ", which is the ratio of the two partly-standardised ratios $A / B$ and $B / C$. A movement of the $\mathrm{L} 3$ vein away from the $\mathrm{L} 4$ vein would increase $\mathrm{D}$. A comparison of $\operatorname{tg}^{C}$ Short with each of the other lines is summarised in table 2. In $\operatorname{tg}^{C}$ Short, in which the L2 vein is missing, the distance between the L3 and L4 veins

TABLE 2

Comparisons of standardised vein separations $(D)$. The $P$-values are given for $\mathrm{t}$-tests between each pair of lines shown in table 1

$\begin{array}{lccccc}\operatorname{tg} C \text { Short } & <0.001 & <0.001 & <0.001 & <0.001 & >0.05 \\ \operatorname{tg} C \text { unselected } & - & >0.05 & >0.05 & >0.05 & <0.001 \\ \text { ri Short } & - & - & >0.05 & >0.05 & <0.001 \\ \text { OKI } & - & - & - & >0.05 & <0.001 \\ \text { Canton-S } & - & - & - & - & <0.001\end{array}$

is significantly larger than that in $\operatorname{tg}^{C}$ unselected and in the control lines, in which the $\mathrm{L} 2$ vein is present and complete. The single exception is the wild type strain Oregon- $R$, in which the arrangement of veins differs in many ways from the other wild control lines.

The suggestion of minor inter-strain differences led us to see whether there were better ways of measuring vein placement in relation to wing 
shape. Wing shape can be characterised roughly by the ratio of wing width to wing length. With the exception of $\operatorname{tg}^{C}$, which is relatively longer than the others, the wings in our study do not differ significantly in shape (table 3 ). Thus, we can standardise vein placement (essentially vein angles and spacing)

\section{TABLE 3}

Vein spacing standardised to wing width. Distances are measured from a standard base line as illustrated in fig. 2

\begin{tabular}{|c|c|c|c|c|c|}
\hline \multirow[b]{2}{*}{ Stock } & \multirow[b]{2}{*}{$\mathrm{N}$} & \multirow{2}{*}{$\frac{\text { Width }}{\text { Length }}$} & \multicolumn{3}{|c|}{ Standardised mean spacing } \\
\hline & & & L2 & L3 & L4 \\
\hline $\operatorname{tg}^{C}$ Short & 20 & $0.667 \pm 0.0145$ & 一 & $0 \cdot 618$ & $0 \cdot 385$ \\
\hline $\operatorname{tg}^{C}$ unselected & 19 & $0.656 \pm 0.0139$ & $0 \cdot 939$ & 0.590 & $0 \cdot 380$ \\
\hline$O K 1$ & 20 & $0.664 \pm 0.0248$ & 0.920 & 0.563 & $0 \cdot 361$ \\
\hline Oregon- $R$ & 20 & $0.671 \pm 0.0192$ & 0.938 & $0 \cdot 586$ & $0 \cdot 353$ \\
\hline
\end{tabular}

in relation to wing width (fig. $2 b$ ). A measurement of the placement of the L2, L3, and L4 veins in relation to wing width confirms the observations obtained from the previous methods of scoring. The L3 vein in $\operatorname{tg}^{C}$ Short is shifted significantly toward the anterior margin $\left(\operatorname{tg}^{C}\right.$ Short versus $O K 1$, $\mathrm{P}<0.001 ; \operatorname{tg}^{C}$ Short versus $\operatorname{tg}^{C}$ unselected, $0.05>\mathrm{P}>0.01 ; \operatorname{tg}^{C}$ Short versus Oregon- $R, 0.01>\mathrm{P}>0.001)$, in an apparent compensation for the absence of the L2 vein.

When compared to the wild type controls, the L4 vein in $\operatorname{tg}^{C}$ Short is also shifted slightly in an anterior direction, though there is no difference between the relative placement of the $\mathrm{L} 4$ vein in $\operatorname{tg}^{C}$ Short and in unselected. This confirms that the L3 vein is the principal element involved in this example of pattern compensation.

Because of the complexities associated with measuring spacing from wing photographs, we wanted to measure inter-vein distances using an independent morphological character. We had noted earlier that the number of marginal hairs between the L3 and L4 veins (fig. 1) is relatively constant within strains (Schaefer and Thompson, 1977). These marginal hair counts are a measure of distance based upon inter-vein cell number. Data from such counts (table 4) confirm the relationships found using other measures of vein spacing and show a pattern compensation in $\operatorname{tg}^{C}$ when the L2 vein is absent. The standardised hair counts are largest in $\operatorname{tg}^{C}$ Short $\left(\operatorname{tg}^{C}\right.$ Short compared to control lines: $O K 1, \mathrm{t}_{92}=13 \cdot 1, \mathrm{P}<0.001 ;$ Canton- $S, \mathrm{t}_{94}=13.5$,

TABLE 4

Means and standard deviations for counts of hairs along the margin of the wing between the L3 and L4 veins

\begin{tabular}{|c|c|c|c|c|}
\hline Stock & $\mathrm{N}$ & $\begin{array}{l}\text { Hair number } \\
\qquad(\mathrm{h})\end{array}$ & $\begin{array}{l}\text { Wing length } \\
\text { (1) }\end{array}$ & Mean of $h / 1$ \\
\hline $\operatorname{tg}^{C}$ Short & 47 & $36 \cdot 6 \pm 1 \cdot 11$ & $48 \cdot 4 \pm 1 \cdot 09$ & $0.757 \pm 0.029$ \\
\hline $\operatorname{tg} C$ unselected & 40 & $34 \cdot 3 \pm 3 \cdot 54$ & $50 \cdot 8 \pm 2 \cdot 13$ & $0.676 \pm 0.033$ \\
\hline$O K 1$ & 47 & $33 \cdot 7 \pm 1 \cdot 75$ & $53 \cdot 1 \pm 2 \cdot 44$ & $0.637 \pm 0.055$ \\
\hline Canton-S & 49 & $31 \cdot 3 \pm 1 \cdot 56$ & $46 \cdot 8 \pm 1 \cdot 77$ & $0.669 \pm 0.035$ \\
\hline Oregon- $R$ & 49 & $35 \cdot 8 \pm 1.73$ & $48 \cdot 6 \pm 2 \cdot 39$ & $0.737 \pm 0.038$ \\
\hline $\begin{array}{l}\text { Heterozygous progeny of } \\
\operatorname{tg}^{C} \text { Short } \times \text { OK } 1\end{array}$ & 48 & $36 \cdot 6 \pm 1 \cdot 65$ & $52 \cdot 9 \pm 0.88$ & $0.692 \pm 0.032$ \\
\hline
\end{tabular}


$\mathrm{P}<0.001 ;$ Oregon $R, \mathrm{t}_{93}=3.1,0.01>\mathrm{P}>0.001 ; \operatorname{tg}^{C}$ unselected, $\mathrm{t}_{85}=11.9$, $\mathrm{P}<0.001$ ), though there is clear interstrain variation. This confirms the intriguing minor strain differences in overall vein arrangement that might be uncovered by further strain comparisons.

\section{Discussion}

(i) General observations on vein determination

In developmental genetics, as in population genetics, artificial selection can be a powerful experimental tool. Minor gene effects can be accumulated to modify a pattern by small, regular steps. This, in turn, allows one to concentrate upon the changing interactions among pattern elements and their natural range of variation. When applied to the Drosophila wing, the techniques of quantitative genetics and of mosaic analysis have merged to give us an interesting insight into some of the properties of the vein pattern. The wing mosaic studies have confirmed that vein pattern is, to a large extent, independent of cell lineage, while the precision of quantitative genetics has uncovered inter-vein compensations that may provide a useful clue to the mechanisms of vein placement.

The veins are relatively evenly spaced over the wing, and spacing appears to vary with the size (especially width) of the wing. One is struck by the fact that the veins are remarkably straight and one pair in particular, the distal L3 and L4, are essentially parallel. This arrangement is disrupted, however, when a gap occurs in the more posterior of the two (e.g., the L4 gap in $c i$ ). Displacement of parts of a vein can also occur, as when the missing distal segment of the L2 in $r i$ Short is modified by artificial selection for longer veins (fig. 1h). Displacement of the entire L3 vein in $\operatorname{tg}^{C}$ has been described here in detail.

It is, therefore, clear that any model of vein formation must take into account these two observations: (1) the general independence of cell lineage and final vein placement, and (2) the interdependence among veins in their shape and spacing.

\section{(ii) A model of vein determination}

There appear to be two classes of vein mutations: those that have single vein-specific effects and those that affect all longitudinal veins or inter-vein areas (Thompson, 1974). These two groups can be modified by the same polygenic modifiers to a limited extent and, thus, appear to represent biochemical pathways that interact directly in the formation of vein. However, the mutant groups also differ in interesting ways. Vein-specific mutants generally have subterminal vein gaps that are sensitive to temperature variation, while vein-general mutants that show loss of vein usually have terminal gaps and are commonly not temperature sensitive. The veingeneral mutants with gaps and those with extra vein fragments appear to be opposite expressions of the same developmental process (Waddington, 1940).

Prior to the formation of the veins, the wing is a single tissue-layer balloon with a dorsal and a ventral surface (Waddington, 1940). Beginning at both the proximal and distal wing margins, veins are laid down over a period of between 9 and 10 hours as this balloon begins to collapse. The crossveins are the remnants of the central cavity. A key event in the formation of 
veins appears to be the presence or absence of intercellular processes connecting the two tissue layers. Where these processes are formed, the layers come to lie close together; where they are inhibited, a space remains. Later, extra chitin is deposited in all areas in which a space separates the dorsal and ventral surfaces to form the veins.

To account for the two classes of vein mutants and their effects upon wing development, our model assumes a minimum of two components. The first is a region-specific component in which individual vein competence is coded and which can be affected by individual vein mutants (e.g., $c i$ and $r i$ ). This is perhaps equivalent to a prepattern. The second is a component that affects the expression of veins in general. Mutants in this category either influence the expression of all longitudinal veins (e.g., ve) or add fragments to all parts of the wing $(e . g ., p x)$. When the interaction between these two components has exceeded a threshold, the intercellular processes connecting dorsal and ventral wing surfaces are inhibited, and a space or "vein" is formed.

One can visualise the model in more specific terms by considering the following hypothetical case. First, the wing might be viewed as a mosaic of longitudinal regions, each of which has the capacity to produce a specific modulator. Second, an enzyme affecting the formation of intercellular processes is assumed to diffuse from points along the margin of the wing between the vein (modulator) competent regions (fig. 3). Where the enzyme diffusing from adjacent points and a particular modulator reach a critical threshold, the formation of intercellular processes is inhibited. If the inhibition reaction ties up the modulator-enzyme complex, this would produce a sink for the reaction. The idea of a continuing reaction and sink is totally consistent with the observation that veins are formed, then erased, in both general and vein-specific mutants (Waddington, 1940). The overall result is a series of well-spaced veins.

Where a regional deficiency in modulator production occurs, a gap would appear in a specific vein. For example, in a mutant such as $c i$, we might hypothesise a localised deficiency in the production of the L4 modulator. Because of a change in the reaction sink, this deficiency will change the point at which the reaction for nearby veins exceeds the threshold. Consequently, the L3 bends towards the gap in the L4. In the case of $\operatorname{tg}^{C}$, veins spread to fill the space left by the removal of the $\mathrm{L} 2$ vein.

We would like to emphasise that this is but one possible case, and we are not proposing that this is the precise mechanism involved. Rather, this example simply serves to illustrate the type of interaction between components that is most consistent with the observed changes in vein pattern in response to the alteration or elimination of elements. In addition, it is important to realise that this model implies a mechanism for developmental homeostasis, in that it automatically provides a compensation for differences in wing size (as between sexes) and in wing shape.

Garcia-Bellido (1977) has shown that the expression of many vein mutants is autonomous. This is quite consistent with a model such as the one outlined above, in that there are a number of components required for mutant or vein expression that are regionalised within the wing. Autonomous expression simply implies that the "enzyme-modulator" complex influences the formation of intercellular connections on a cell by cell basis. Although it provides a basis for designing future experiments, the primary 

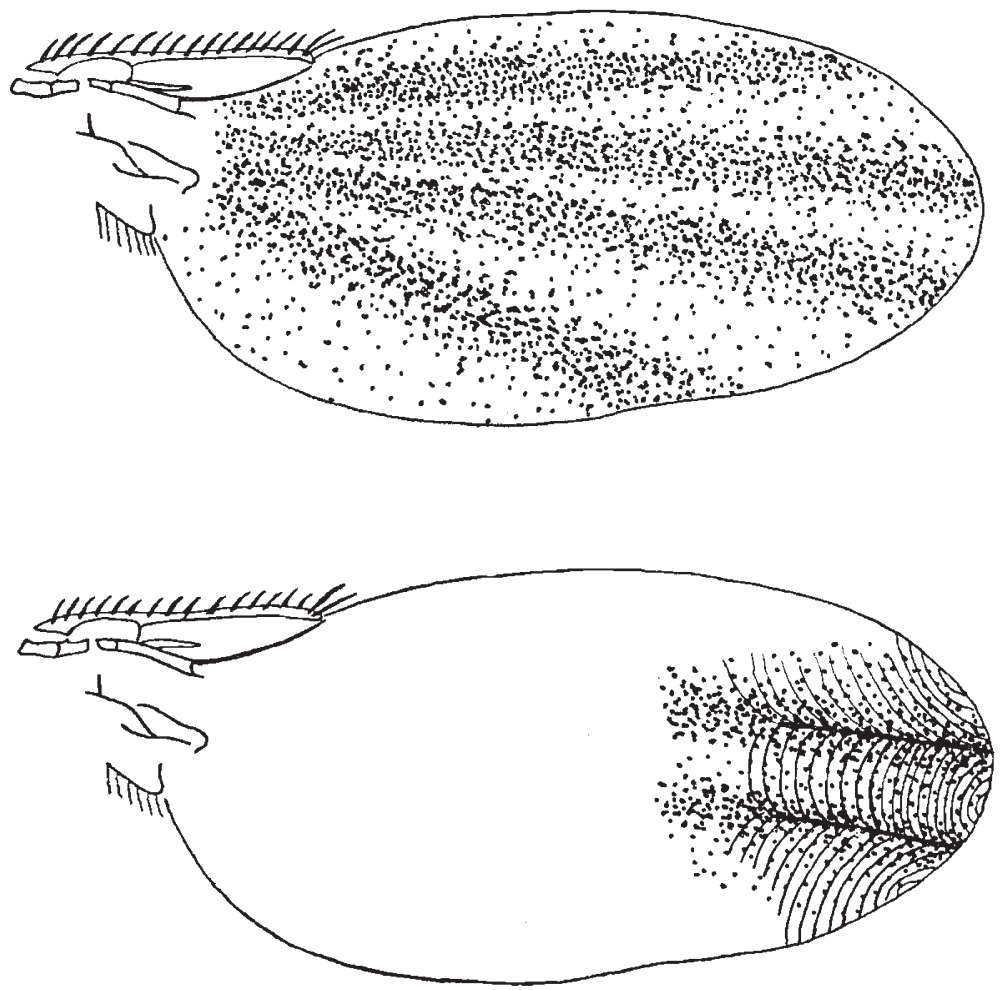

FIG. 3.-Summary of a general model to explain the determination of vein placement. The top figure represents a series of regions of individual vein competence; the bottom figure represents the interaction of diffusion gradients. See text for explanation.

contribution of our study is not the model we have described. Rather, it is the suggestion that one can view the formation of a particular pattern of elements (the wing veins) as the product of self-regulating interactions, with or without autonomous regions of competence. This flexibility is seen as developmental homeostasis that compensates for variations in size and shape of the wing without requiring specific cell determination early in development.

Acknowledgments.-We thank Dr Peter Lawrence and Ms Marian Cothran for reading the manuscript and Ms Marla Chess for drawing fig. 2. The work was supported by D.H.E.W.-N.I.H. grant 1-R01-GM-24809-01.

\section{REFERENGES}

BRYANT, P. J. 1970. Cell lineage relationships in the imaginal wing disc of Drosophila melanogaster. Developmental Biology, 22, 389-411.

Carlson, J. H. 1966. Genetics of an L2 venation mutant in Drosophila melanogaster. I. Mode of inheritance and expression. Ohio J. Sci., 66, 340-346.

CARLSON, J. H. 1970. Genetics of an L2 venation mutant in Drosophila melanogaster. II. Pattern effects. Ohio J. Sci., 70, 365-371.

garcIa-BELliDo, A. 1975. Genetic control of wing disc development in Drosophila. In: Cell Patterning. Ciba Foundation Symposium No. 29, 161-182. 
GARCIA-BELLIDO, A. 1977. Inductive mechanisms in the process of wing vein formation in Drosophila. Wilhelm Roux's Archives, 182, 93-106.

GARCIA-BELlido, A., AND RIPOLL, P. 1978. Cell lincage and differentiation in Drosophila. In Genetic Mosaics and Cell Differentiation, ed. W. J. Gehrung, pp. 119-156. SpringerVerlag, New York.

GARCIA-BELlido, A., RIPOLL, P., AND MORATA, G. 1973. Developmental compartmentalization of the wing disk of Drosophila. Nature New Biology, 245, 251-253.

KAURFMAN, s. A. 1977. Chemical patterns, compartments, and a binary epigenetic code in Drosophila. Amer. Zool., 17, 631-648.

LAWRENCE, P. A. 1973. The development of spatial pattern in the integument of insects. In Developmental Systems: Insects, ed. S. J. Counce and C. H. Waddington, vol. 2, pp. I57209. Academic Press, New York.

Lindsley, D. L., AND GRELl, E. H. 1968. Genetic Variations of Drosophila melanogaster. Carnegie Institution of Washington, Publ. No. 627.

stern, C. 1968. Genetic Mosaics and Other Essays. Harvard Univ. Press, Cambridge, Mass. SGHAEFER, G. B., AND THOMPSON, J. N., JR. 1977. Mutant influences upon vein patterns in Drosophila. Bios, 49, 103-109.

SGHAEFER, G. B., TONEY, J. v., AND THOMPSON, J. N., JR. 1978. Evidence supporting a diffusion model for the origin of wing vein patterns in Drosophila melanogaster. Genetics, 88, s86-s87.

THOMpson, J. N., JR. 1973. A new suppressor of veinlet and comments on two other vein mutants. Drosophila Information Service, 50, 59.

thompson, J. N., JR. 1974. Studies on the nature and function of polygenic loci in Drosophila. II. The subthreshold wing vein pattern revealed in selection experiments. Heredity, 33, $389-401$.

ToKUnaga, c. 1978. Genetic mosaic studies of pattern formation in Drosophila melanogaster, with special reference to the prepattern hypothesis. In Genetic Mosaics and Cell Differentiation, ed. W. J. Gehring, pp. 157-204. Springer-Verlag, New York.

waddington, C. H. 1940. The genetic control of wing development in Drosophila. F. Genet., $41,75-139$.

WOLPERT, L. 1969. Positional information and the spatial pattern of cellular differentiation. 7. Theoret. Biol., 25, 1-47.

WOODRUFF, R. C., AND THOMPSON, J. N., JR. 1977. An analysis of spontaneous recombination in Drosophila melanogaster males: Isolation and characterisation of male recombination lines. Heredity, 38, 291-307. 